\title{
DESIGN STUDIES FOR AN EXPERIMENT TO MEASURE ENERGY SPREAD EVOLUTION THROUGH A SOLENOIDAL FOCUSING SYSTEM*
}

\author{
Y. Cui ${ }^{\dagger}$, A. Valfells, S. Bernal, M. Reiser, P.G. O'Shea, R.A. Kishek, V. Yun, N. Rahimi \\ Institute for Research in Electronics and Applied Physics, College Park, Maryland 20742
}

\begin{abstract}
The University of Maryland Electron Ring (UMER) [See invited paper by P.G. O'Shea] requires a detailed knowledge of the energy spread of the beam. The evolution of energy spread in space-charge dominated beams is not well understood. There are several sources that cause the energy spread growth, such as the Boersch effect and the longitudinal-longitudinal relaxation effect. According to theoretical analysis, the energy spread should be a few eV, however this has not been verified. We plan to use a cylindrical energy analyzer, which has resolution of $0.5 \mathrm{eV}$, to measure the evolution of energy spread along a $2 \mathrm{~m}$ transport line with a $3-5 \mathrm{keV}, 70-135$ $\mathrm{mA}$ beam. The test beamline will have 3 short solenoids for matching into a long solenoid and 1 short solenoid for matching into the diagnostic chamber. Two energy analyzers will be used to measure energy spread at different positions in the transport line.
\end{abstract}

\section{INTRODUCTION TO LONGITUDINAL ENERGY SPREAD EVOLUTION}

Theoretical understanding of energy spread evolution in charged-particle beams, from emission to equilibrium, is still not well understood $[1,2]$. There are three main physical processes involved: Cooling due to acceleration; longitudinal-longitudinal effects, and the Boersch effect.

When the particles come off of the emitter the beam is in thermal equilibrium (i.e. the transverse and longitudinal temperatures are equal). This implies that the energy spread in the transverse direction is the same as that in the longitudinal direction. When the particles are accelerated the energy spread remains constant, but the longitudinal velocity spread is decreased and hence the longitudinal temperature decreases. Equation (1.1) describes the relation between the initial longitudinal temperature, $\mathrm{T}_{\| \mathrm{i}}$, and the longitudinal temperature immediately after acceleration, $\mathrm{T}_{\| \mathrm{f}}$.

$$
k_{B} T_{\| f}=\frac{\left(k_{B} T_{\| i}\right)^{2}}{2 q V_{0}}
$$

For instance a beam that comes off of the emitter with a temperature of $0.1 \mathrm{eV}$ is cooled to a temperature of $10^{-6}$ eV.

Another effect of accelerating the beam is to increase the intraparticle spacing in the axial direction. Since the acceleration time is short compared to the charged particle

\footnotetext{
*Work supported by the Department of Energy

${ }^{\dagger}$ Email: cuiyp@glue.umd.edu
}

plasma period this increase occurs mostly after acceleration. The transfer mechanism is via Coulomb collisions. This is known as the longitudinal-longitudinal effect. Equation (1.2) gives the energy spread after acceleration:

$$
\Delta \tilde{E}_{\| f}=\left[\frac{C}{\pi \varepsilon_{0}} q n^{1 / 3} q V_{0}+2 q V_{0} k_{B} T_{\|_{f}}\right]^{1 / 2}
$$

The first term on the right-hand side of (1.2) is due to the longitudinal-longitudinal effect, with $\mathrm{C}$ being a constant on the order of unity.

After acceleration the beam will not be in thermal equilibrium, with the transverse temperature higher than the longitudinal temperature. Various effects such as Coulomb collisions, instabilities, etc. will drive the beam towards equilibrium lowering the transverse temperature and increasing the longitudinal temperature. This is known as the Boersch effect [3]. The longitudinal heating of the beam will manifest itself as an increase in the longitudinal energy spread of the beam, since we have the following relation:

$$
\Delta \tilde{E}=\sqrt{2 q V_{0} k_{B} T_{\|}}
$$

So the energy spread scales like the square root of the temperature. As was mentioned above one of the mechanisms leading to equilibrium is Coulomb collisions. If one assumes that this is the only mechanism [4] at play one gets the following equation for the temporal development of the longitudinal temperature:

$$
T_{\|}=\frac{2}{3} T_{\perp i}\left(1-e^{-3 t / \tau_{\text {eff }}}\right)
$$

where $\tau_{\text {eff }}$, the effective temperature relaxation time, is dependent on the particle density of the beam and the initial transverse temperature, $\mathrm{T}_{\perp \mathrm{i}}$. As an example we note that for particles coming off of the emitting surface with a temperature of $0.1 \mathrm{eV}$, cooling down through acceleration and subsequently heating up to a temperature of $0.067 \mathrm{eV}$ via the Boersch effect the longitudinal energy spread will go from $0.1 \mathrm{eV}$ at the emitter to $36 \mathrm{eV}$ at equilibrium.

\section{ORGANIZATION OF EXPERIMENT}

To measure the energy spread growth, an experiment will be performed along a $2.2 \mathrm{~m}$ transport line measuring the longitudinal energy spread at two points along that line. We use thermionic triode gun with a variable accelerating potential ranging from $3-5 \mathrm{kV}$ and current ranging from $70-135 \mathrm{~mA}$. The beamline will have 3 short solenoids for matching into a long solenoid and 1 short 
$221 \mathrm{~cm}$

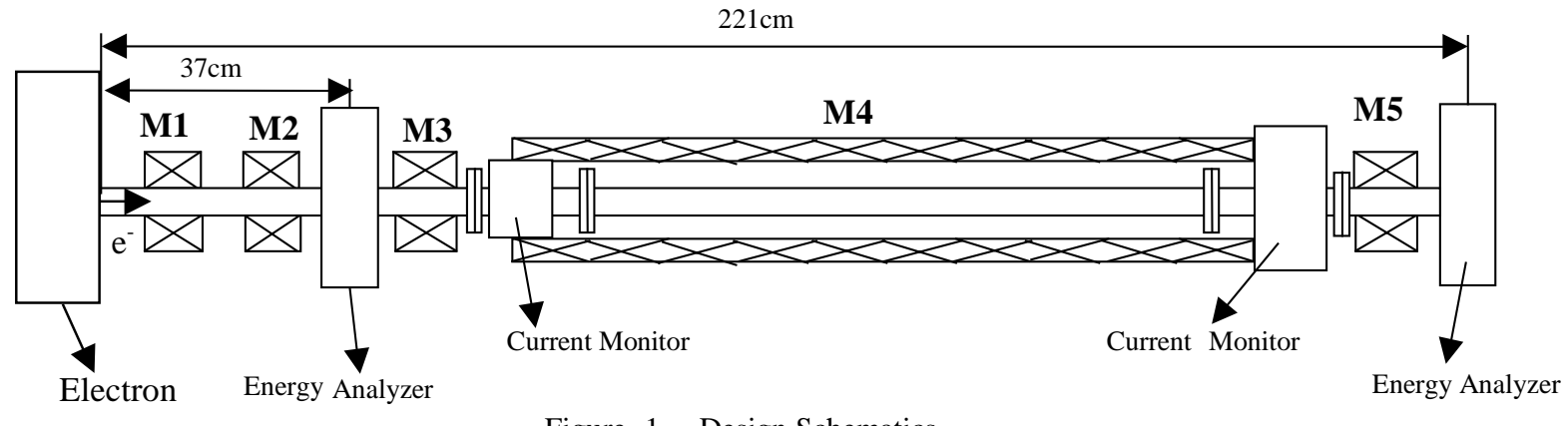

Figure 1. Design Schematics

solenoid for matching into a diagnostic chamber. One energy analyzer is situated between the second and third short solenoid and may be retracted from the path of the beam. This analyzer is placed at a beam-waist. The second energy analyzer is located at the end of the beamline. The distance between the two analyzers is $184 \mathrm{~cm}$. Figure 1. shows a schematic drawing of the experiment where M1, M2, M3, and M5 are short solenoids. The long solenoid, M4, serves as a uniform focusing channel. Two current monitors are included to monitor possible beam loss. Using this setup we hope to be able to measure the variation of the energy spread for different accelerating voltages. The effective lengths of these solenoids are in the Table1.

Table1:The effective lengths of solenoids

\begin{tabular}{|l|l|l|l|l|l|}
\hline $\begin{array}{l}\text { effective } \\
\text { length }(\mathrm{cm})\end{array}$ & M1 & M2 & M3 & M4 & M5 \\
\cline { 2 - 6 } & 4.34 & 4.24 & 7.28 & 130.8 & 7.08 \\
\hline
\end{tabular}

The short solenoids are all alike with an inner diameter of $7.6 \mathrm{~cm}$. Each one is controlled by a separate DC power supply for ease of matching the beam under different conditions. The long solenoid (M4) is $138.7 \mathrm{~cm}$ long. It is made of copper windings on an aluminum tube with diameter of $11.5 \mathrm{~cm}$. There is an iron tube at the outside of the copper windings to restrict the field lines.

From WARP code [5] simulation, Figure 2 shows the total magnetic field on axis needed to match the $3 \mathrm{keV}$

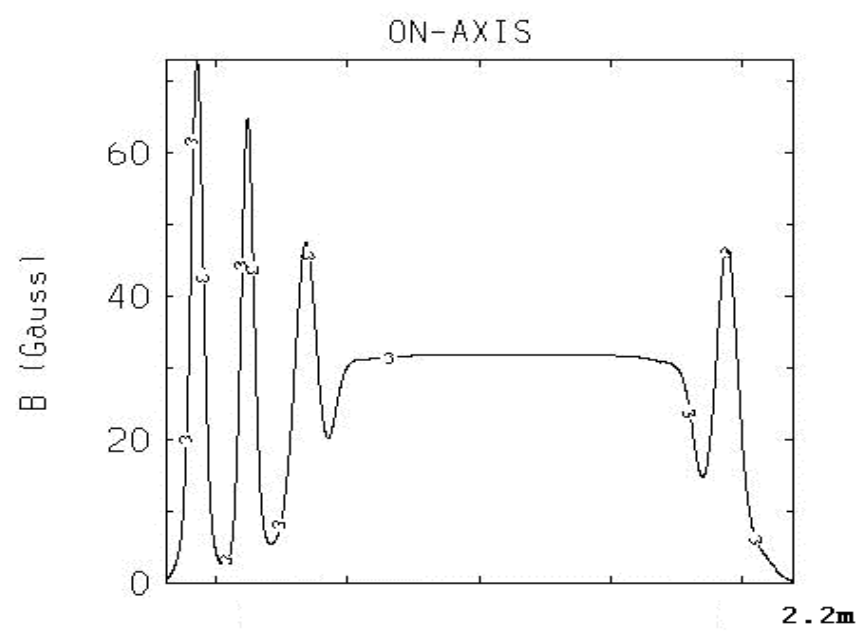

Figure2.Applied magnetic field for $3 \mathrm{KeV}$ Beam

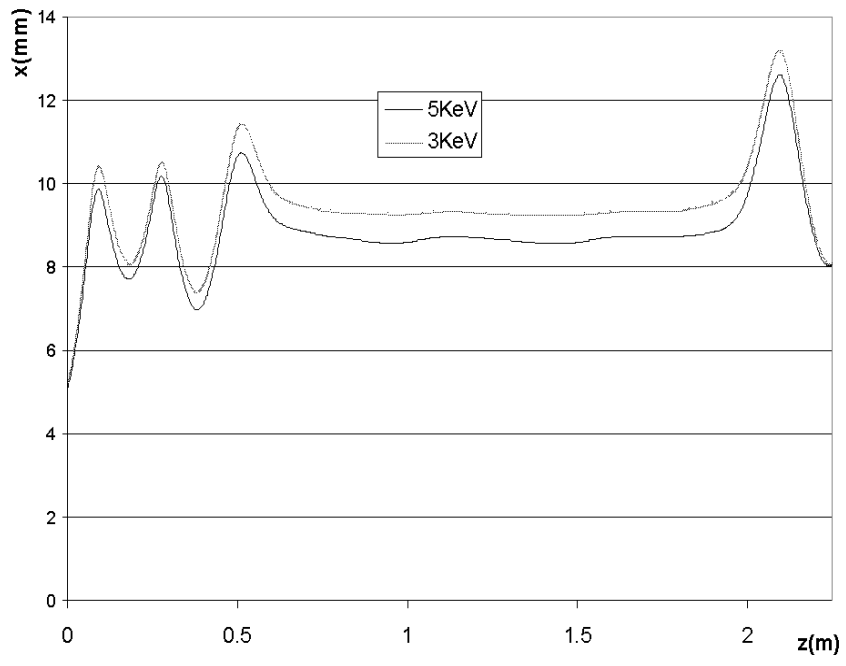

Figure3. Simulation Envelope for $3 \mathrm{KeV}$ and $5 \mathrm{KeV}$

beam. Figure 3 shows the matched envelope for the $3 \mathrm{keV}$ and $5 \mathrm{keV}$ beams.

Let us now make an estimate of the energy spread we expect to see in our experiment. Using measurements of the beam current and beam radius at $3 \mathrm{kV}$ and $5 \mathrm{kV}$ accelerating potentials one may come up with an estimate of the relaxation time, and of the increased energy spread due to longitudinal-longitudinal effects. Then we used Equations (1.2) and (1.4) to estimate the energy spread at various points along the $\mathrm{z}$-axis for beam energies of $3 \mathrm{keV}$ and $5 \mathrm{keV}$, also assuming a cathode temperature of $0.1 \mathrm{eV}$. The results of these calculations are shown in Figure 4.

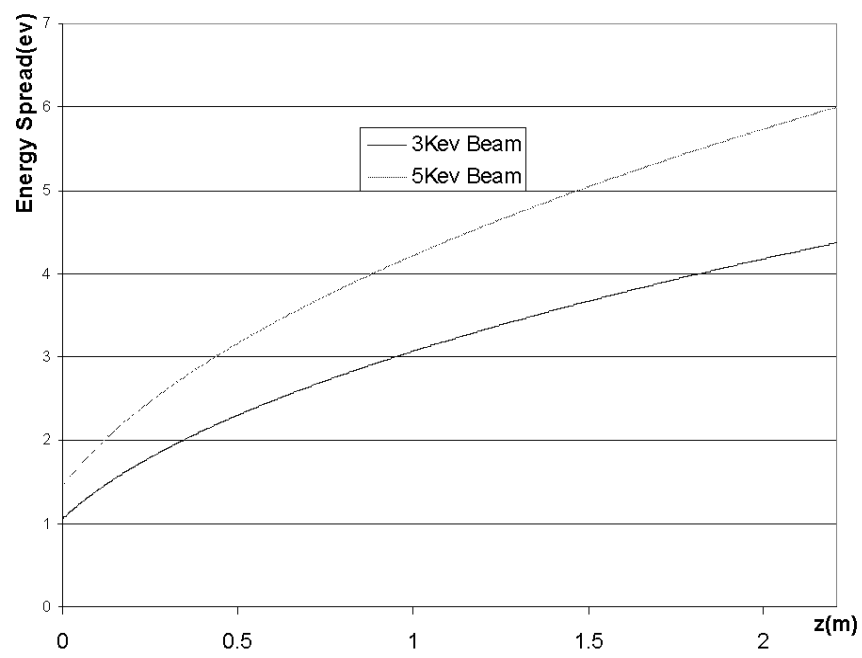

Figure 4.Theory Calculation For Energy Spread Growth 
The first energy analyzer is positioned at the beam waist roughly $37 \mathrm{~cm}$ from the anode, and the second is $2.2 \mathrm{~m}$ from the anode. From the theoretical calculation, we have Table 2.

Table 2: Energy spread for 2 different positions

\begin{tabular}{|l|l|l|}
\hline $\mathrm{z}$ & $0.37 \mathrm{~m}$ & $2.2 \mathrm{~m}$ \\
\hline $3 \mathrm{keV}$ & $2.05 \mathrm{eV}$ & $4.36 \mathrm{eV}$ \\
\hline $5 \mathrm{keV}$ & $2.83 \mathrm{eV}$ & $6.00 \mathrm{eV}$ \\
\hline
\end{tabular}

To measure such small energy spreads the energy analyzers must have good resolution. To this end we use a cylindrical energy analyzer, developed previously [6], that has a smaller inherent energy spread than a parallel electrode analyzer. The goal of such a design is to focus incoming electrons on to the collector in such a manner that they come as close as possible to impinging perpendicularly upon the retarding grid. In this energy analyzer the focusing electrode and the retarding grid are electrically connected. We have altered this design somewhat so that we may have the focusing electrode at a separate potential from the retarding grid. Figure 5. shows a cross-sectional view of the new designed analyzer. Diameter of the hole through which electrons enter is $2 \mathrm{~mm}$. Simulations of this design using the SIMION[7] indicate that the resolution of the energy analyzer may be improved significantly, from $8 \mathrm{eV}$ to $0.5 \mathrm{eV}$. Figure 6 . shows the simulation results for the energy analyzer for a $10 \mathrm{keV}$ beam with a $0.5^{\circ}$ spread in the incident angles of the electrons. Figure 7. shows corresponding results using the new design with a focusing potential of $10.13 \mathrm{kV}$.

\section{FUTURE WORK}

The next step is to finish building and testing the new

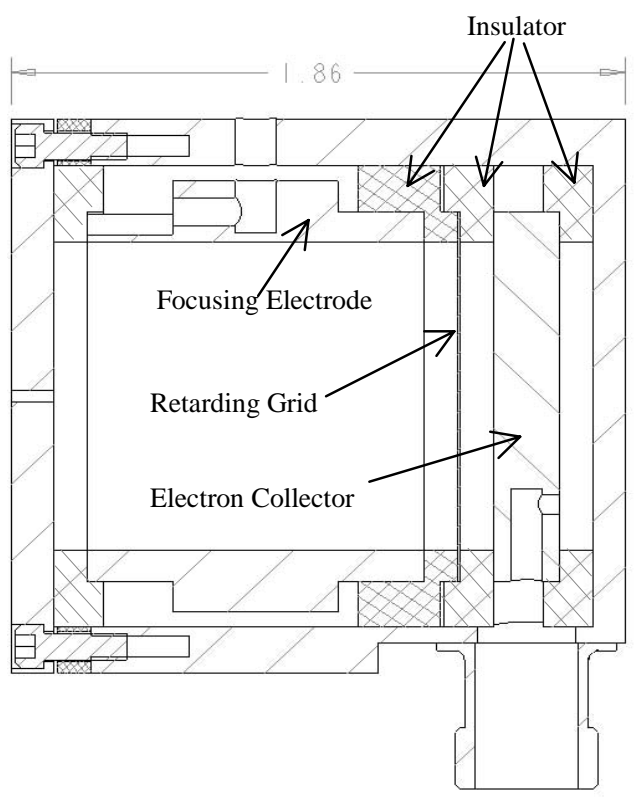

Figure5.cross-sectional view of new designed energy analyzer

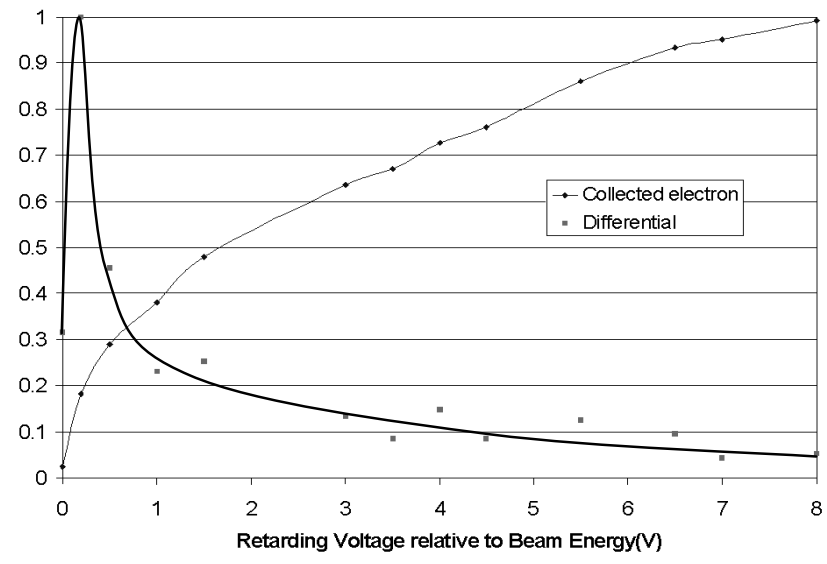

Figure6.Previous Energy Analyzer Resolution (10keV Beam, By Applying same Focusing

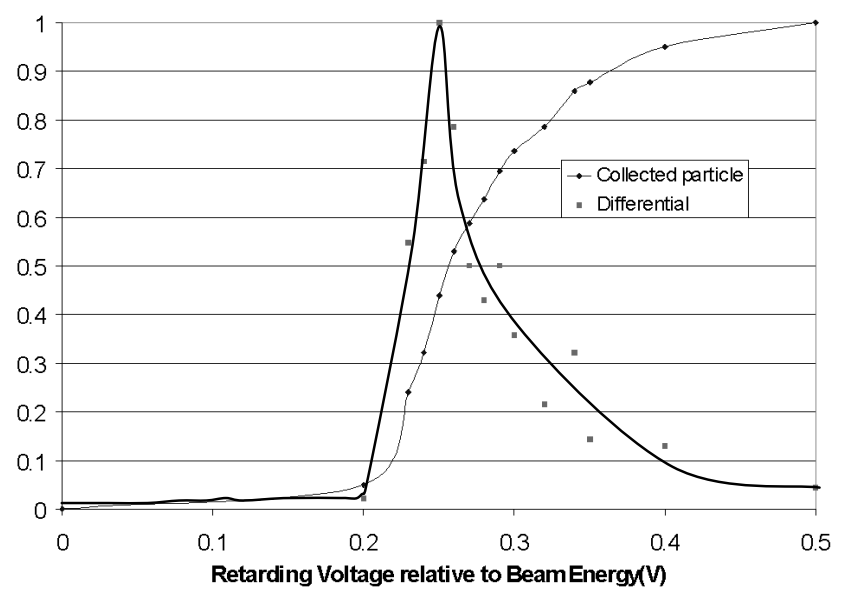

Figure7.Energy Analyzer Resolution Improvement Result (10keV Beam, By Applying 10.13kVFocusing Voltage )

energy analyzer, as well as to implement a design for using a single power supply to the focusing electrode and retarding grid. This will minimize any effects of variations in the high-voltage.

After this we will continue our experiment on the energy-spread evolution. We will also do simulations using the WARP code [5] to compare with. In this way we hope to determine what mechanisms are of major importance to the equipartitioning process.

\section{REFERENCES}

[1] M. Reiser, Theory and Design of Charged Particle Beams (John Wiley \& Sons, Inc, New York,1994).

[2] V.I.Kudelainen, V.A.Lebedev,etc Temperature relaxation in a magnetized electron beam,

Sov.Phys.JETP,Dec.1982.

[3] H.Boersch, Z.Phys. 139,115 (1954)

[4] S. Ichimaru and M. N. Rosenbluth, Phys. Fluids 13, 2778 (1970)

[5] WARP Menu

[6] Yun Zou, Ph.D. Dissertation, Department of Electrical Engineering, University of Maryland, 2000

[7] SIMION Menu 